\title{
Clinical, functional and genetic characterization of Sixteen Patients Suffering from Chronic Granulomatous Disease variants - Identification of Twelve Novel Mutations in CYBB
}

Marie josé STASIA ${ }^{1}$, Michelle Mollin² ${ }^{2}$ Sylvain Beaumel ${ }^{2}$, Benedicte Vigne ${ }^{2}$, Julie Brault ${ }^{2}$, Nathalie Roux-Buisson ${ }^{2}$, John Rendu ${ }^{2}$, Vincent Barlogis ${ }^{3}$, Gaud Catho ${ }^{4}$, Claire Dumeril ${ }^{5}$, Fanny Fouyssac ${ }^{6}$, Delphine Monnier ${ }^{7}$, Virginie Gandemer ${ }^{7}$, Matthieu Revest ${ }^{7}$, Jean-Paul Brion $^{8}$, Cecile Bost-Bru ${ }^{9}$, Eric Jeziorski1 ${ }^{10}$, Laurence Eitenschenck ${ }^{5}$, Clemence Jarrasse $^{5}$, and Stephanie Drillon Haus ${ }^{11}$

${ }^{1}$ Université Grenoble Alpes

${ }^{2}$ Centre Hospitalier Universitaire Grenoble Alpes Institut de Biologie et de Pathologie

${ }^{3} \mathrm{AP}-\mathrm{HM}$

${ }^{4}$ Hospices Civils de Lyon

${ }^{5}$ Centre Hospitalier Annecy Genevois

${ }^{6}$ Centre Hospitalier Universitaire de Nancy

${ }^{7}$ Centre Hospitalier Universitaire de Rennes

${ }^{8}$ Centre Hospitalier Universitaire Grenoble Alpes

${ }^{9}$ Centre Hospitalier Universitaire Grenoble Alpes Pole Couple Enfant

${ }^{10}$ Centre Hospitalier Universitaire de Montpellier

${ }^{11}$ Centre Hospitalier Universitaire de Strasbourg

July 1, 2020

\begin{abstract}
Chronic Granulomatous Disease (CGD) is a rare inherited disorder in which phagocytes lack NADPH oxidase activity. The most common form is the X-linked CGD (X-CGD), caused by mutations in the CYBB gene. Clinical, functional and genetic characterizations of 16 CGD cases of male patients and their relatives were done. We classified them as suffering from different variants of CGD (X910, X91- or X91+) according to NOX2 expression and NADPH oxidase activity in neutrophils. Twelve mutations were novel (10 X910-CGD and 2 X91- -CGD). One X910-CGD was due to a new and extremely rare double missense mutation Thr208Arg-Thr503Ile. We investigated the pathological impact of each single using stable transfection of each mutated cDNA in the NOX2 knock-out PLB-985 cell line. Both mutations leading to X91-CGD were also novel; one deletion -67delT was localized in the promoter region of CYBB, the second one c.253-1879A $>$ G mutation activates a splicing donor site, which unveils a cryptic acceptor site, leading to the inclusion of a 124-nucleotide pseudo-exon between exons 3 and 4 and responsible for the partial loss of NOX2 expression. Both X91-CGD mutations were characterized by a low cytochrome b558 expression and a faint NADPH oxidase activity. The functional impact of new missense mutations is discussed in the context of a new 3D-model of the dehydrogenase domain of NOX2. Our study demonstrates that low NADPH oxidase activity found in both X91-CGD patients correlates with mild clinical forms of CGD whereas X910-CGD and X91+-CGD cases remain the most clinically severe forms.
\end{abstract}

\section{Hosted file}


Main manuscript Stasia MJ2020CEI.pdf available at https://authorea.com/users/338749/articles/ 465204-clinical-functional-and-genetic-characterization-of-sixteen-patients-sufferingfrom-chronic-granulomatous-disease-variants-identification-of-twelve-novel-mutations-in$\mathrm{cybb}$

a

\section{$\operatorname{CGD}^{\circ}{ }^{\circ} \mathrm{X}$}
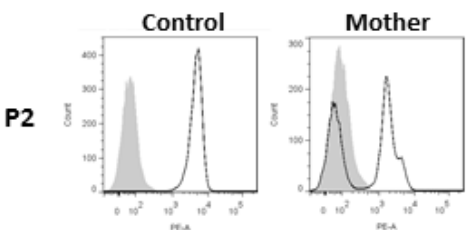

P3
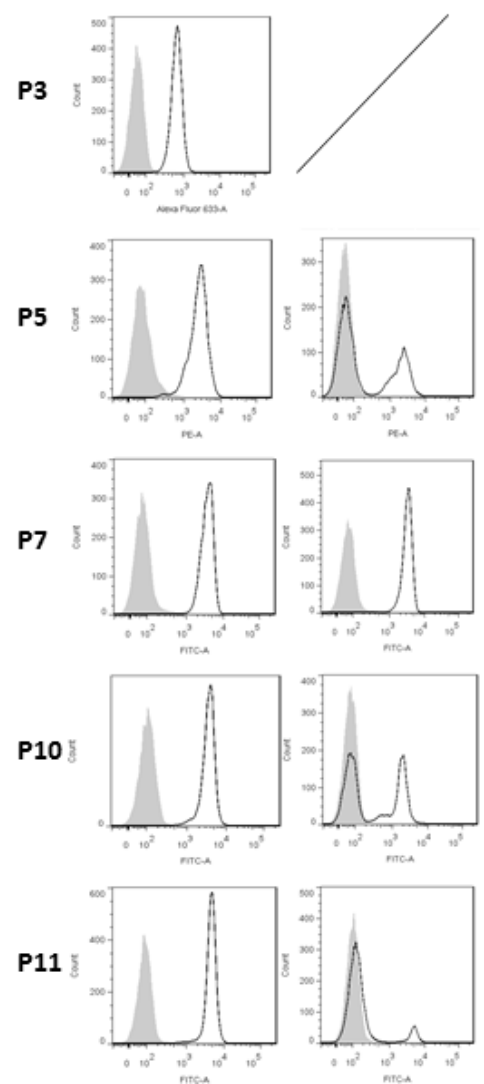

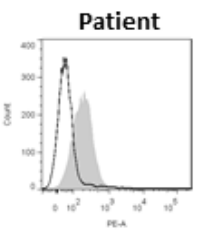

b

$$
\text { P12 - CGD91 }{ }^{\circ} \mathrm{X}
$$
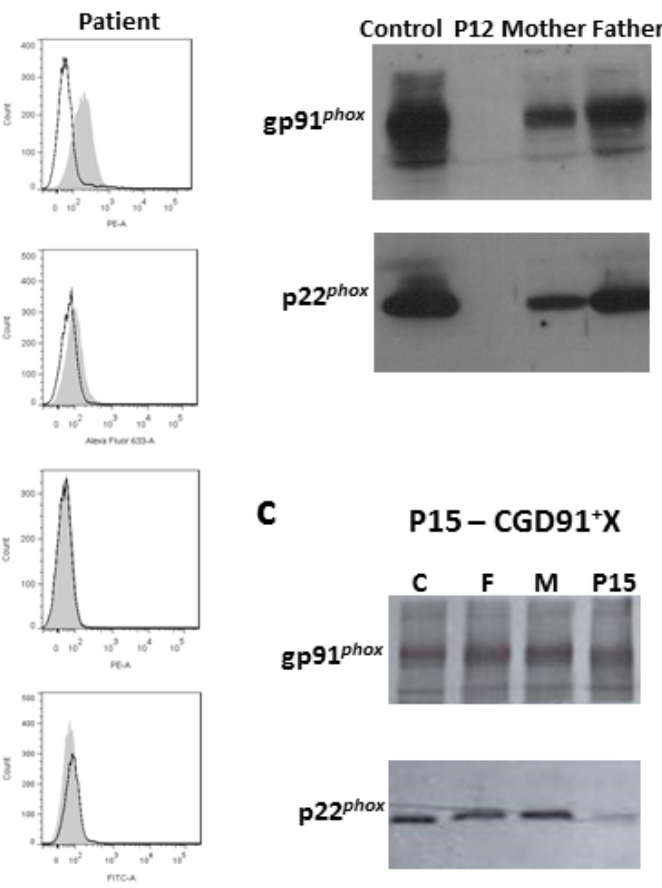

C
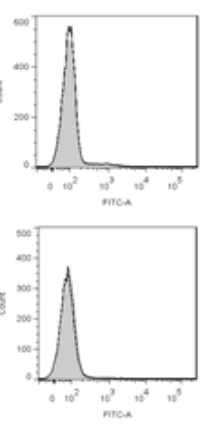
a

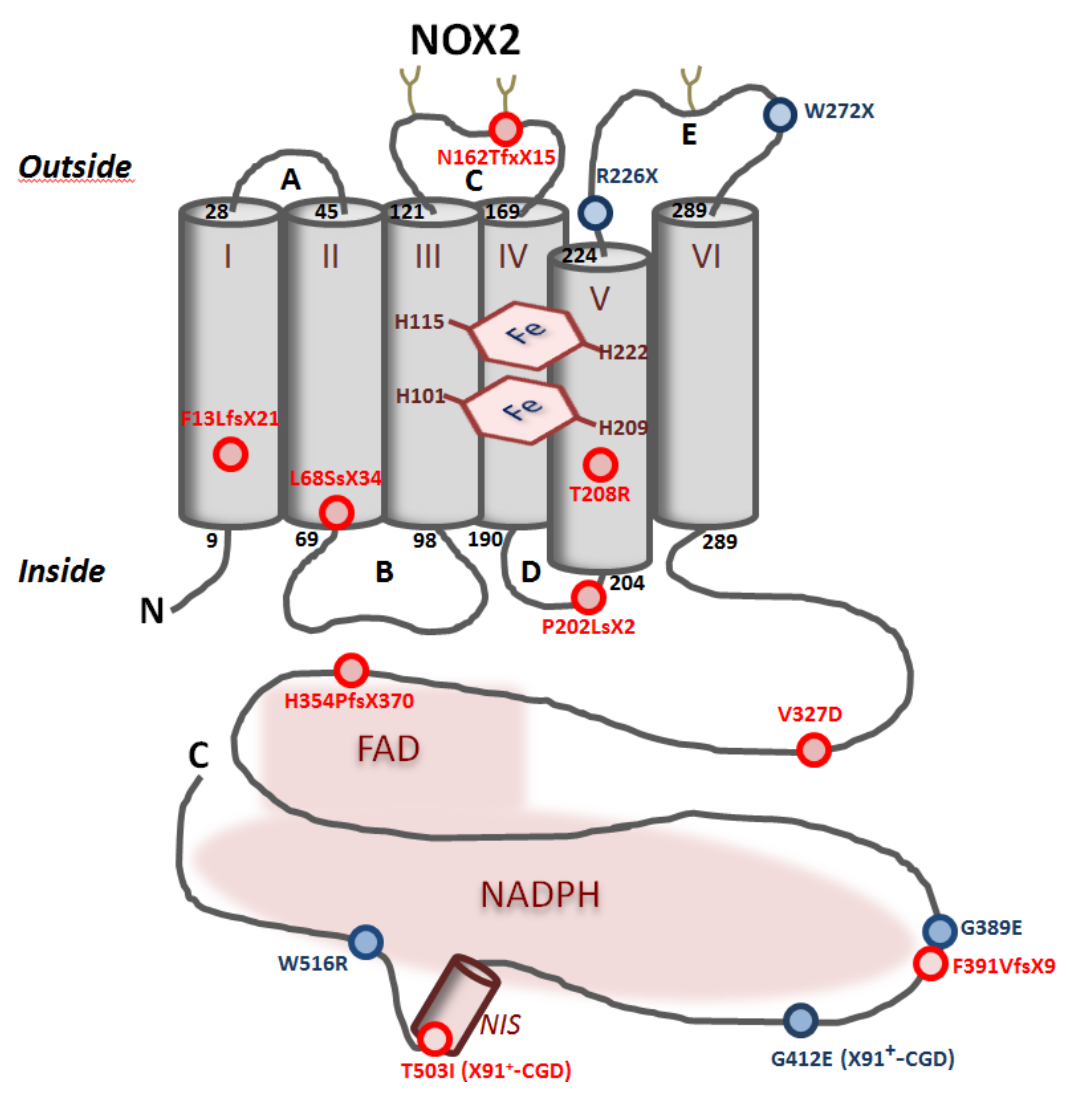


b
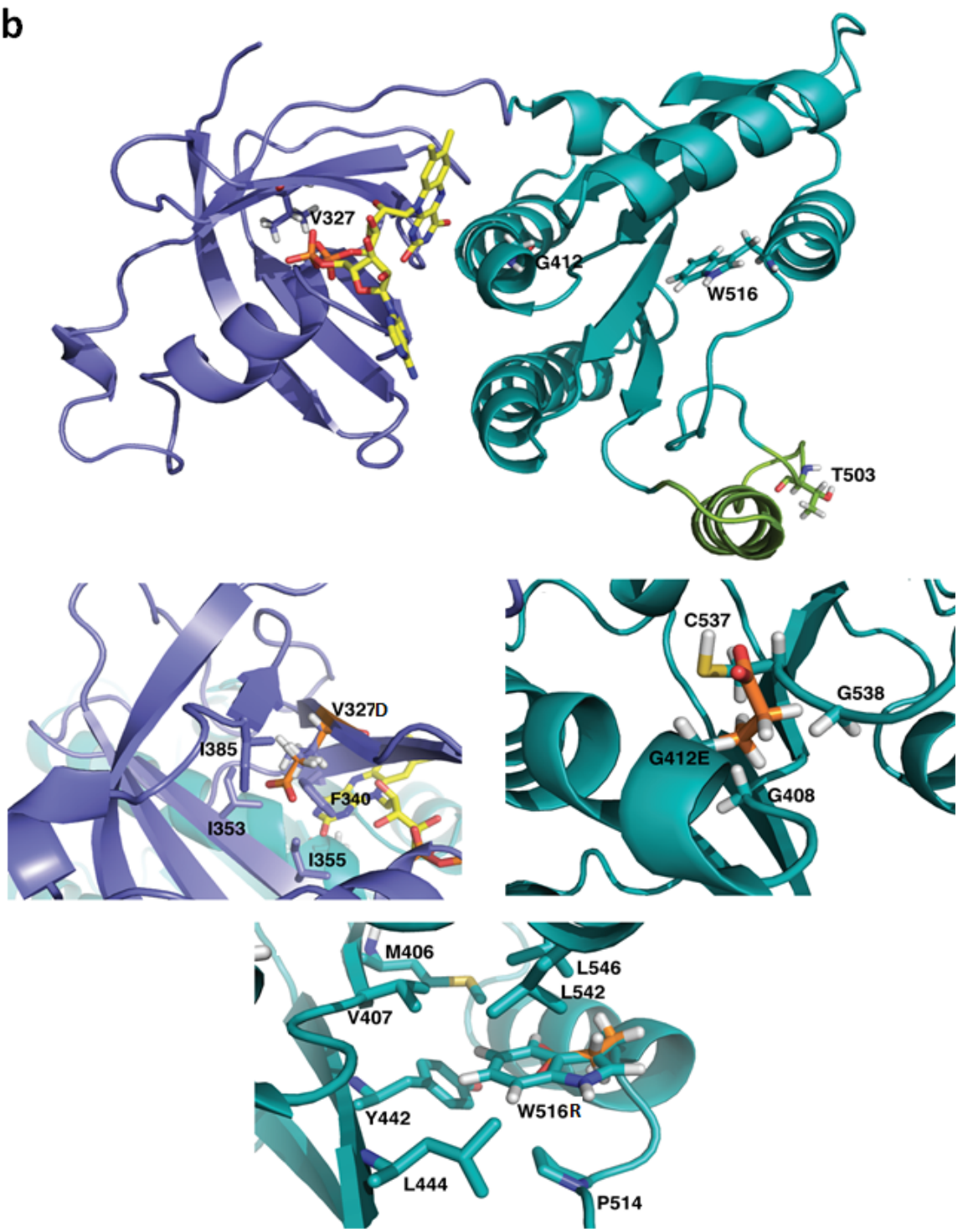
a

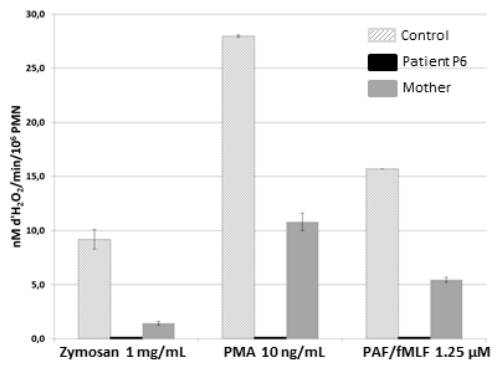

d
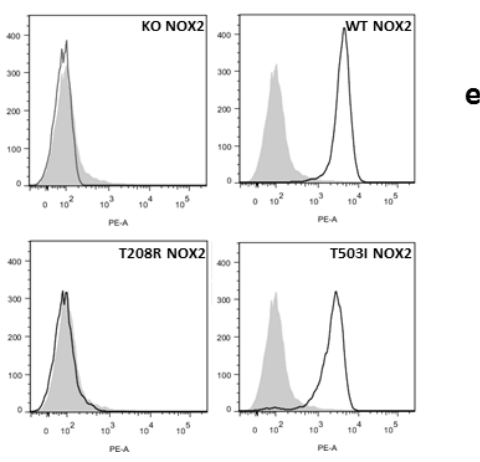

b
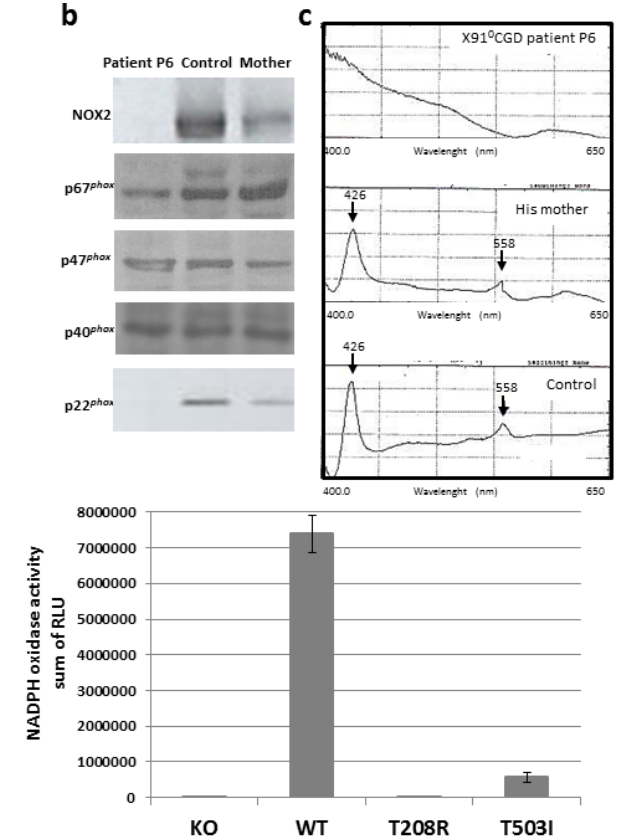
a

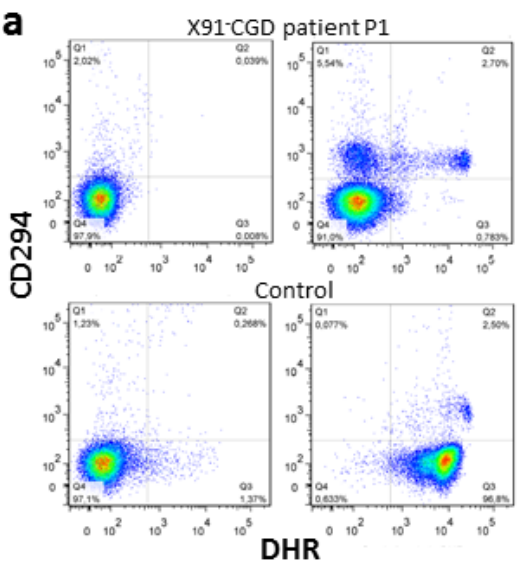

C
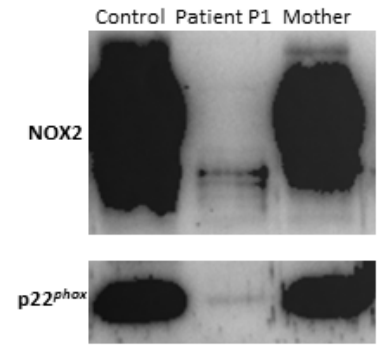

e

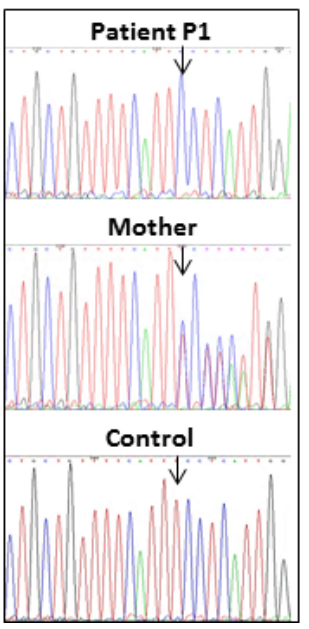

b
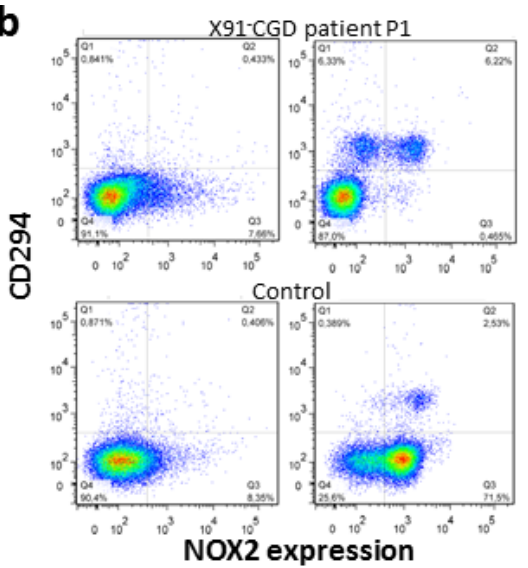

d
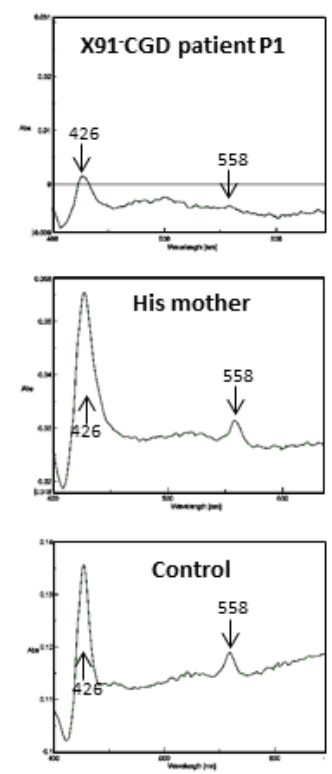

f

cagttgaccaatgattattagccaatttctgataaaagaaa aggaaaccgattgccccagggctgctgttttca $\Pi(T)$ cctc -67delT

attggaagaagaagcatagtatagaagaaaggcaaacaca acacattcaacctctgccacc ATG GGG...(exon 1) 
a
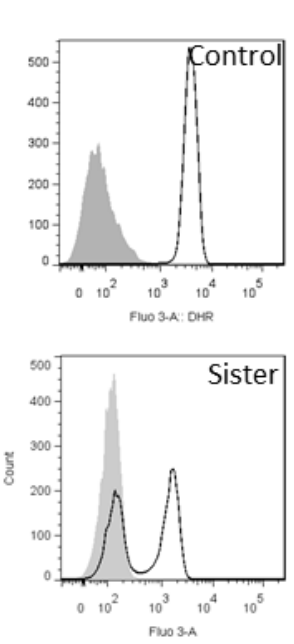

d
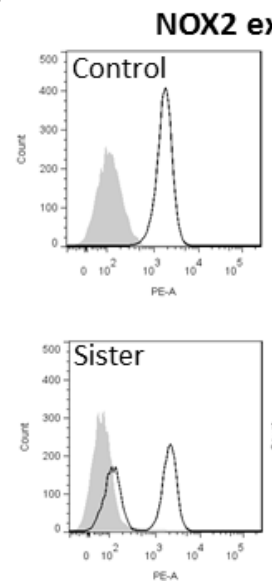

DHR
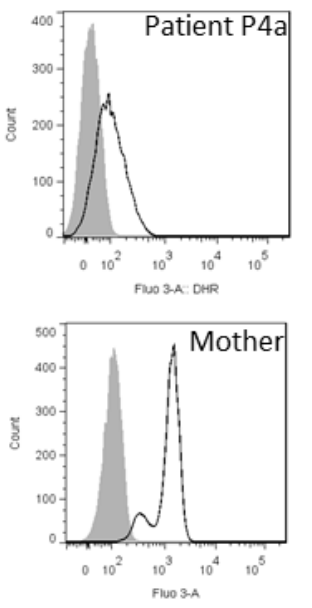

b

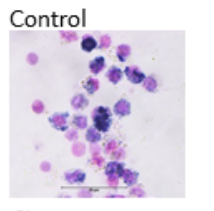

Sister

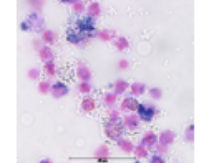

C

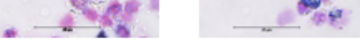

Mother Patient P4a Control

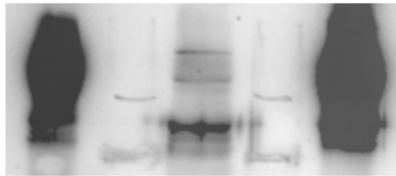

p22 $2^{\text {phox }}$

e p22 ${ }^{\text {phox }}$ expression
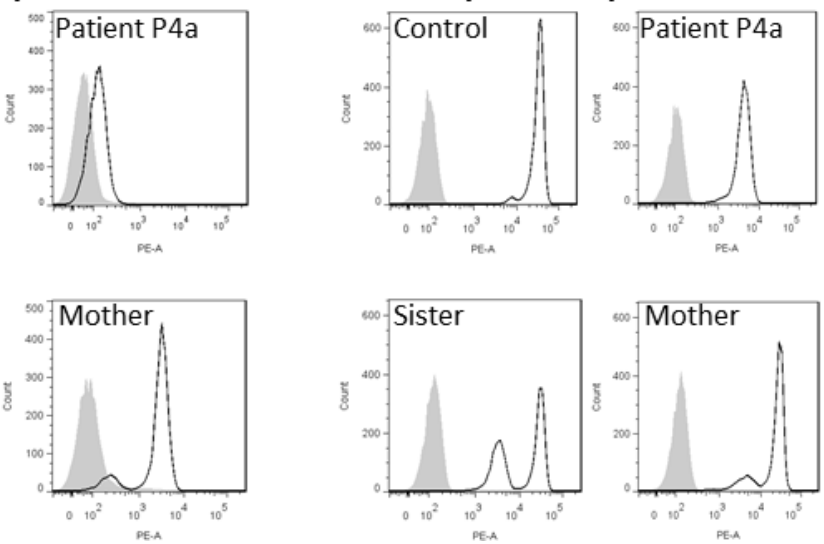
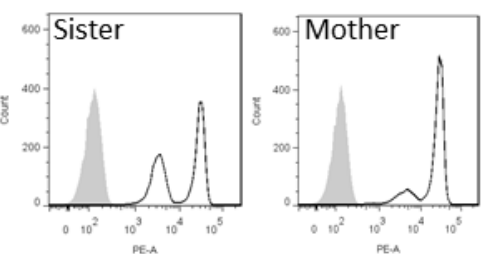
f

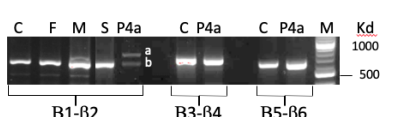

B1- $-\beta 2$

B3- $-\beta 4 \quad$ B5'- $-\beta 6$

c. 252

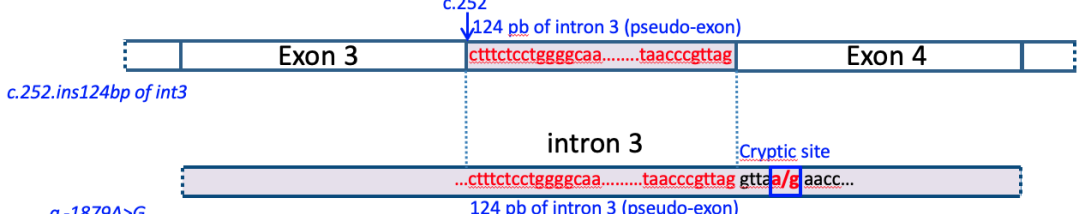

MGNWAVNEGL SIFVILVWLG LNVFLFVWYY RVYDIPPKFFYTRKLL

MGNWAVNEGL SIFVILVWLG LNVFLFVWYY RVYDIPPKFFYTRKLL

GSALALARAPAACLNFNCMLILLPVCRNLLSFLRGSSACCSTRVRRQL

GSALALARAPAACLNFNCMLILLPVCRNLLSFLRGSSALSPGALSKSQG

DRNLTFHKMVAWMIAL HSAIHTIAHL...

IDVLRASLRAALSOSWMAVGGSTOP 